are now so largely used. All these things are full of danger, being liable to take fire on exposure to a very moderate degree of heat. A woman kneeling in front of, but by no means very near to, a clear red fire, and having an imitation tortoiseshell comb in her hair, on the top of her head, became conscious of a smell of burning and a very disagreeable vapour around her, and on putting up her hand found that her head was on fire. She poured water over it and got it out, but not before a great deal of her hair was burnt. In another case the patient was seated in a drawing room before a fire which, though burning well, was not unusually strong, when she found her head enveloped in smoke and flame from the ignition of a comb amongst her hair, and before the flames could be extinguished' she had a portion of her scalp destroyed over an area of perhaps four by one and a-half inches. The burn took many months to heal, and resulted in the total destruction of the hair over the injured area. Professor Ogston, with the assistance of Professor Japp, has made a series of experiments with the object of ascertaining the temperature at which celluloid will take fire when exposed to heat, and he finds that, although in the case of good, well-made celluloid, the temperature at which it will inflame is stated to be $383^{\circ}$ Fahr., a far lower temperature will induce this effect with some of the common specimens which are employed in the manufacture of the articles mentioned above. For example, a portion of the comb which caused the accident in one of the cases was placed in contact with the steel of a hat pin, the steel likewise touching the thermometer, and the whole being placed in front of a fire. The moment the thermometer registered $200^{\circ} \mathrm{Fahr}$. the celluloid ignited. Another portion of the same comb was enveloped, with a thermometer, in a small lock of the black hair of the patient, and exposed to the radiant heat of the fire. The celluloid in this case took fire when the thermometer marked $180^{\circ} \mathrm{Fahr}$. In another case, in which a child's fair hair was used, the celluloid burnt at $167^{\circ} \mathrm{Fahr}$. Such cases are sufficient to show how extremely dangerous a head decoration is this inflammable material, and how very important it is that some measures should be taken to prevent the use for the purpose of badly manufactured celluloid, which evidently is liable to catch fire at temperatures far below that which is normal in the case of the properly prepared article. Even with properly manufactured celluloid, although, no doubt, the danger is less, women would be well advised not to decorate their heads with articles made of such an inflammable substance, while certainly for boys and men, who smoke and use matches, to surround their necks and wrists with false cuffs and collars which are liable to go off like fireworks if touched by a spark, is the height of folly.

$$
1 \text { Lancet, Feb. } 22 .
$$

\section{WHAT TO DO WITH THE APPENDIX.}

THE interest of the last meeting of the Clinical Society centred principally on the question whether or not the appendix should be removed when operating upon cases of appendicitis with abscess formation in the acute stage. Mr. Barling strongly advised that unless the appendix could be removed with ease it should be left alone. In advising this course he insisted upon the safety with which pus could be evacuated if the removal of the appendix were not insisted upon, and the rarity with which recurrence took place under such circumstances. He said that the abscess should be attacked as soon as the diagnosis of suppuration was made, and that in making this diagnosis the presence of leucocytosis was of much significance. The statistics he gave strongly bore out the propriety of the treatment which he had employed. Of 140 cases of appendicitis operated on in the acute stage, 74 had been cases of localised suppuration in the right iliac fossa or immediately adjacent to it, and in 46 of the 74 cases the abscess, though well shut off by adhesions, had not come forward so as to be adherent to the abdominal parietes, and thus the operation involved opening the peritoneal cavity. In these 74 cases there were 2 deaths. The appenaix was only removed 25 times, and in 49 cases it was not removed. Mr. F. C. Wallis read a paper on the treatment of intraperitoneal abscess of the appendix in which he stated that his practice was to remove the appendix. The point of his paper however rather lay in his insistance on the fragile condition of the abscess in those cases among young people in which, although shut off by adhesions from the general peritoneal cavity, the abscess was in no way adherent to the abdominal parietes. In these cases it was necessary to open the general peritoneal cavity before the abscess could be dealt with, and great care was necessary to prevent the pus under tension in the abscess sac from escaping into the general cavity of the peritoneum. Packing with Gamgee sponges and gradual evacuation was recommended. Mr. Arthur Barker was in favour of removing the appendix at the time of the primary operation if it could be done easily, and if not then by a secondary operation in the quiescent interval. Mr. James Berry thought it was inadvisable to remove the appendix in all cases of suppurative appendicitis. In fact, even in regard to incising the abscess he seemed to advocate a waiting policy; waiting, that is, until adhesions had taken place and making a small incision. Mr. W. G. Spencer considered that it was advisable to remove the appendix at the time of the first operation. Take it altogether the general opinion seemed to be that the appendix was better removed if-always if-this could be done easily, but that it was better to let it alone than to hunt for it, which was much what Mr. Barling had said, although there was some difference of opinion as to how far the hunt should be carried, and also as to the suggested harmlessness of an appendix when left unremoved.

\section{THE SUBCUTANEOUS INJECTION OF QUININE.}

THE superiority of the subcutaneous injection of quinine over its administration by the mouth in certain cases of malaria has long been recognised, although many causes have intervened to prevent the universal adoption of this method. Among these objections may be mentioned the difficulty of dissolving the ordinary sulphate of quinine without adding so much acid as to cause the solution to become very irritating and painful, and, in hot climates, the fear, which seems a very real fear in some places, of causing tetanus. Much would seem to depend upon the preparation employed, the salt usually recommended being the acid hydrochloride, 
which is very soluble. Dr. Ferguson, ${ }^{1}$ of Cheltenham, however, endorses the recommendation of the bi- or acid hydrobromate of quinine, as being far superior to other preparations for this purpose. The examples given by Dr. Ferguson are very striking, half a dozen subcutaneous injections having been sufficient to cure many cases of long standing which had already been subjected to treatment by quinine in other forms without effect. In explaining the superior efficacy of hypodermic injections as compared with administration by the mouth, he refers to the rapid excretion of the drug by way of the kidneys as being the cause of the apparent inactivity of quinine in some cases. The excretion of quinine through the kidneys begins a few minutes after a dose has been given, and it is this fact that explains the superior results from its subcutaneous use. When slowly absorbed through disordered stomach and intestinal membranes it is excreted nearly as fast as it is absorbed and never reaches in the blood a strength sufficient to destroy the malaria plasmodia. On the other hand, when subcutaneously used, its maximum and effective strength is probably reached within half an hour. As to method, Dr. Ferguson injects subcutaneously 3 grains of quinine bi-hydrobromate dissolved in 20 minims of pure warm water. He first injects it under the skin of the upper arm, then under that of the thighs, then under the skin of the abdomen, or at the top of the chest or between the scapulæ. Six injections on alternate days are usually required in a serious case. He keeps a syringe for the purpose, and uses it for nothing else, disinfecting it and the patient's skin with strong carbolic lotion, carefully disinfecting his own hands, and sterilising the needle in the flame of a spirit lamp. The solution of quinine is sterilised when first made, but he has not himself boiled it each time before using, although he now recommends that this should be done. With such precautions, he is convinced that there need be no fear of tetanus, and further he is convinced that there are few or no cases of malarial fever that will resist six subcutaneous injections of 3 grains each of quinine bi-hydrobromate.

1 Brit. Med. Jour., Feb. 22.

\section{EPISTAXIS IN RHEUMATISM IN CHILDREN.}

A $\mathrm{T}$ a recent meeting of the Harveian Society Dr. Sidney Phillips drew attention to the frequency with which epistaxis is found in association with evidence of rheumatism in some form or another, an association which he regarded as too frequent to be put down to mere coincidence. After detailing a series of cases he said that the urine had been examined in all of them and was found to be free from albumin. There was no local nasal disease, and no cause could be found for the epistaxis except that there were evidences of a rheumatic diathesis. Many other cases could have been brought forward to illustrate the same point. In some of them the epistaxis came on with an attack of acute rheumatism, in others epistaxis alternated with attacks of articular inflammation, or was preceded by them, and in some cases it occurred in connection with chorea, which was presumably rheumatic. As is well known synovial effusion into joints and acute articular rheumatism may occur in children without any pain. Hence, with this clue in our minds, the occurrence of an attack of epistaxis may, on the one hand, suggest examination from other rheumatic symptoms, while, on the other hand, the discovery of such symptoms may be taken as explaining a perhaps puzzling occurrence of epistaxis in a child.

\section{PROGRESS IN SURGERY.}

OTOLOGY.-Continued from page 361 .

The Technique of Mastoid Operations.-W. Milligan $^{8}$ alludes to the difficulty there is in the radical operation of efficiently clearing away every focus of disease. The use of burrs or osteotribes is recommended to help to decide if all disease has been removed. Before the introduction of skin grafting some parts of the tympanum after the radical operation often failed to get an epidermal covering, e.g. the inner tympanal wall and the receding antrotympanic angle, and therefore a granulating surface was left. Milligan considers that Ballance's operation, which he has tried repeatedly, has revolutionised the operation for chronic otorrhœa. He suggests that the skin incision be opened up without a general anæsthetic the day before the skin grafting is performed, in order to avoid the oozing of blood during the latter procedure. Another suggestion is to fill the antro-tympanic cavity quite full of normal saline fluid, and float the graft on it, and then rapidly suck out the fluid with a pipette passed through the meatus. Thus the graft is made to sink down closely upon the walls of the cavity it is desired to cover. The writer prefers glass to metal stoppers for pressing home the gold leaf, because they adhere less to the gold leaf. The remains of the posterior meatal wall should be carefully smoothed down lest the grafts be broken there over a sharp edge. The pinna may be slung up to the top of the head by tapes soaked in collodion Panse and Leutert ${ }^{9}$ maintain that reinfection of the cavities operated upon in and about the ears occurs through the Eustachian tube, and they therefore endeavour to shut off the tube by causing the remnant of the membrana tympani to adhere to the promontory. Trautmann ${ }^{10}$ states that galvanocauterisation of the edges of the Eustachian opening in the tympanum, followed by firm tamponading: against the inner tympanic wall, will effect closure. Galvano-cauterisation of the pharyngeal orifice of the tube is ineffectual. Trautmann also favours the formation of a persistent retro-auricular opening after the radical operation to bring about epidermisation. In operating he works backwards to the antrum from the attic and aditus, if he thinks the lateral sinus lies in a far forward position. This is likely to be the case if the mastoid process has a narrow and high form. If the mastoid, however, is flat and wide, the antrum may be opened at once, and the attic exposed by working forwards. He divides the cutaneous meatal canal posteriorly and horizontally, so as to form an upper narrow flap and a lower broad one. The tampons used consist of acetate of aluminium gauze or xeroform gauze. These are less irritating to the skin than iodoform gauze. Dusting the wound with orthoform renders the insertion of tampons less painful. The retroauricular opening should not be closed for 